A N N A L E S Annales de Bretagne et des Pays de l'Ouest

\title{
Roméo Arbour, Dictionnaire des femmes libraires
} (1470-1870)

Jean Quéniart

\section{(2) OpenEdition}

1 Journals

Édition électronique

URL : http://journals.openedition.org/abpo/1196

DOI : $10.4000 / a b p o .1796$

ISBN : 978-2-7535-1496-6

ISSN : 2108-6443

Éditeur

Presses universitaires de Rennes

Édition imprimée

Date de publication : 20 décembre 2004

Pagination : 156-157

ISBN : 978-2-7535-0082-2

ISSN : 0399-0826

\section{Référence électronique}

Jean Quéniart, «Roméo Arbour, Dictionnaire des femmes libraires (1470-1870) », Annales de Bretagne et des Pays de l'Ouest [En ligne], 111-4 | 2004, mis en ligne le 20 décembre 2006, consulté le 23

septembre 2020. URL : http://journals.openedition.org/abpo/1196 ; DOI : https://doi.org/10.4000/ abpo. 1196 
senté par Renée Leulier tandis qu'à Lorient, où il eut pour tâche de concevoir en 1732 les magasins de la compagnie des Indes, il se reposa sur Louis de SaintPierre (1695-1765) qui s'installa durablement dans la ville (Gérard Le Bouëdec). De même par ses projets, Jacques $\mathrm{V}$ Gabriel contribua à alimenter la propagande royale : à Rennes tout à comme à Bordeaux, les places aménagées servirent d'écrins à la statue du roi. Relais de l'autorité centrale, Jacques V Gabriel a pourtant, de façon paradoxale, contribué à porter les villes de l'ouest atlantique à la pointe de la modernité architecturale avec pour maîtres mots urbanisme planifié et lien entre la commoditas et la voluptas. Alors que Paris ne connaissait encore qu'un urbanisme frôleur et des transformations périphériques, alors que d'autres villes provinciales comme Rouen sortaient lentement de leur gangue moyenâgeuse et décourageaient les initiatives des architectes les plus entreprenants, Rennes et Bordeaux prirent, grâce à lui, un visage nouveau et indiquèrent la voie à suivre.

Le volume se termine par deux communications dues à Alice Thomine et Françoise Hamon qui évoquent la postérité des Gabriel. Rejetés dans la première moitié du XIX ${ }^{\mathrm{e}}$ siècle en tant que symboles de l'Ancien Régime et de la frivolité du XVIII ${ }^{\mathrm{e}}$ siècle, ils connurent un retour en grâce par la suite grâce aux frères Goncourt et firent l'objet d'études de plus en plus nombreuses où ils incarnèrent, en tant que lignée exceptionnelle d'artistes, les vertus de l'hérédité. Associés au triomphe d'un art français autonome dégagé de ses influences italiennes, ils donnèrent naissance, grâce surtout à l'imitation des œuvres de AngeJacques, d'un " néo-Gabriel " qui s'épanouit à Paris mais aussi aux États-Unis au début du Xx ${ }^{\mathrm{e}}$ siècle.

Servi par une présentation impeccable, abondamment illustré, ce volume d'actes, au-delà de son titre quelque peu limité, apporte donc de nombreux éléments à des débats en cours sur les relations Paris-province, sur les pouvoirs de décision dans la France de l'Ouest au XVIII siècle, sur le statut des personnels chargés de mettre en œuvre les politiques adoptées, sur le concept de ville atlantique.

Dominique Le PAGE

ARBOUR, Roméo, Dictionnaire des femmes libraires (1470-1870), Paris, Droz, 2003, 752 p.

750 pages, 6424 notices biographiques appuyées sur un corpus de références précisé ligne à ligne, ces quelques indications montrent d'emblée l'ampleur de ce travail et la sûreté de l'appareil scientifique qui le supporte, tout en facilitant d'autres études à venir. Le dépouillement des fonds de la Bibliothèque nationale, connus de tous les historiens du livre, et pour l'époque contemporaine des Archives nationales, permettent de couvrir sans oubli majeur la totalité du territoire; leur existence témoigne aussi de la surveillance exercée au plus haut niveau par les régimes successifs, à l'exception d'une courte période de la Révolution, sur des professions dont le contrôle était idéologiquement et politiquement indispensable. Il sera utile à chacun de noter, en contrepoint, les pages de l'introduction où les différents sources manuscrites et imprimées font l'objet d'un commentaire critique. La bibliographie est très large, et cependant manque de références à des travaux d'histoire culturelle qui ne portent pas exclusivement sur le livre édité, imprimé ou vendu : les travaux pionniers de Robert Mandrou, par exemple, ne sont pas cités. 
L'ouvrage se veut un instrument de travail pratique, ce qu'il sera sans aucun doute : le classement alphabétique a été dans ce but suivi d'un appendice chronologique, replaçant chaque nom dans son siècle. On peut regretter dans cette perspective l'absence d'un classement géographique, qui eut été fort utile au chercheur local.

L'introduction précise pour chaque époque les conditions juridiques qui limitent ou facilitent pour les femmes l'entrée dans les professions du livre. Sous l'Ancien Régime, seules les veuves peuvent tenir en leur nom imprimerie ou librairie. Outre les lacunes des sources, cette condition par nature temporaire rendrait artificielle toute étude statistique. Pour le XIX ${ }^{\mathrm{e}}$ siècle, il devient au moins possible de dégager de grandes tendances : entre 1840 et 1870, le nombre des femmes à la tête d'une imprimerie ne dépasse qu'exceptionnellement $10 \%$; elles sont beaucoup mieux représentées dans le commerce du livre : toujours proche au minimum de $20 \%$, leur place semble s'accroître sous le Second Empire, pour atteindre $34 \%$ en 1870, terme de l'étude (la période suivante ne permettant pas de distinguer à coup sûr les libraires de femmes qui, tout en ayant un brevet, ne vendent de livres qu'à titre accessoire, pour compléter de maigres ressources).

Certes l'auteur montre comment, au XIX ${ }^{\mathrm{e}}$ siècle, des femmes ont pu trouver dans la librairie des perspectives de promotion sociale; il souligne bien comment, au terme chronologique de son travail, « leur présence dans ces métiers devient normale, comme elle l'était autrefois dans ceux de l'habillement et de l'alimentation ". Mais cette constatation, historiquement importante, d'un changement historique ne vaut guère que pour les dernières années. L'auteur distingue trois niveaux de réussite : les femmes pour qui ce métier n'est qu'un gagne-pain qui ne les sort pas toujours de la pauvreté; celles qui ont connu " une carrière honorable "; " celui des grandes carrières ". Mais, poids numérique mis à part, sontelles en cela bien différentes des hommes? C'est le problème principal que pose un tel ouvrage, qui sera cependant un utile instrument de travail. L'histoire du livre nous semble pouvoir davantage progresser par des études régionales précises : signalons à ce sujet aux lecteurs de cette revue le travail récemment publié de Patricia Sorel, La Révolution du livre et de la presse en Bretagne (1780-1830).

Jean QUENIART

Castagnez, Noëlline, Socialistes en République. Les parlementaires SFIO de la IV République, Presses universitaires de Rennes, coll. " Histoire ", 2004, 414 p.

L'ouvrage est directement tiré de la thèse de doctorat d'histoire que Noëlline Castagnez a soutenue en 2002 à Paris IV sous la direction de Jean-Marie Mayeur, et qui s'intitulait Les parlementaires SFIO de la IVe République. Biographie collective d'une élite militante. Il s'agit d'un modèle d'étude prosopographique, portant sur un corpus de 301 personnes : l'ensemble des hommes et des femmes qui ont exercé, au titre de la SFIO, un ou plusieurs mandats de député ou de sénateur entre 1945 et 1958. Les sources mises en œuvre sont considérables : dossiers individuels des parlementaires, archives internes de la SFIO, papiers personnels de plusieurs dizaines d'intéressés, entretiens avec certains survivants, propagande électorale, discours prononcés à la tribune de l'Assemblée nationale ou du Conseil de la République, etc. L'auteur en tire un portrait de groupe tout à fait passionnant pour qui s'intéresse à l'histoire des partis ou à celle des institutions, croisant en permanence données individuelles et considérations nationales. 